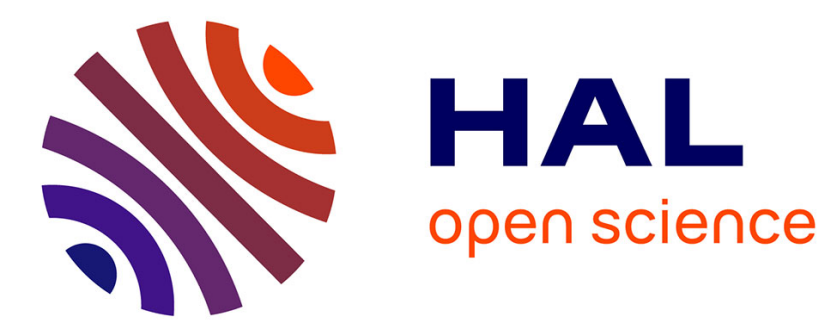

\title{
Albert le Grand écrivain, ou le mystère de la phrase interminable
}

\author{
Dominique Poirel
}

\section{To cite this version:}

Dominique Poirel. Albert le Grand écrivain, ou le mystère de la phrase interminable. Portraits de maîtres offerts à Olga Weijers, dir. Claire ANGOTTI, Monica BRINZEI, Mariken TEEWEN, Porto, 2013 (Textes et études du Moyen Âge, 65), pp.37-62, 2013. halshs-03331484

\section{HAL Id: halshs-03331484 \\ https://shs.hal.science/halshs-03331484}

Submitted on 1 Sep 2021

HAL is a multi-disciplinary open access archive for the deposit and dissemination of scientific research documents, whether they are published or not. The documents may come from teaching and research institutions in France or abroad, or from public or private research centers.
L'archive ouverte pluridisciplinaire HAL, est destinée au dépôt et à la diffusion de documents scientifiques de niveau recherche, publiés ou non, émanant des établissements d'enseignement et de recherche français ou étrangers, des laboratoires publics ou privés. 


\title{
Albert le Grand écrivain ou le mystère de la phrase interminable
}

\author{
Dominique POIREL
}

Quel sens y a-t-il à examiner le style d'un maître de l'université ${ }^{1}$ ? À plus d'un, la démarche paraîtra saugrenue, non sans raison. La méthode scolastique, dont Olga Weijers a si bien analysé les procédures et outils intellectuels, se distingue en effet par deux traits qui semblent à première vue limiter l'intérêt d'une étude de ce genre : d'abord, en ce qu'elle tend à formaliser et à généraliser une sorte de langage commun des maître et des étudiants, grâce à quoi chaque artien, chaque juriste, chaque théologien peut comprendre son confrère d'Oxford, de Bologne ou de Paris et débattre avec lui. L'uniformisation des modes de recherche et d'enseignement, de discussion et d'écriture, est donc un trait essentiel de cette méthode scolastique. D'autre part, la logicisation du discours universitaire conduit à réduire la part de la rhétorique dans la plupart des écrits intellectuels, hormis bien sûr certains textes comme les préfaces ou la prédication. Une certaine indifférence à la beauté sensible du discours est alors le revers de l'extrême importance accordée à la correction formelle de la pensée. Quand on se propose, non de persuader, mais de convaincre, quand on rêve plus ou moins d'une langue abstraite et parfaite, dans laquelle les mots seraient adéquats, les énoncés univoques, les arguments impeccables, la parole doit être bien moins éloquente que transparente. Il semble donc qu'il n'y ait guère de profit à étudier le style chez des auteurs qui, le plus souvent, ne s'en souciaient pas dans leurs propres écrits.

Si cette remarque s'étend à l'ensemble des maîtres universitaires, elle vaut a fortiori pour un docteur comme Albert le Grand. Ce dernier, en effet, semble s'être désintéressé non seulement de l'élégance de sa phrase, mais encore de sa régularité ou même de sa plus élémentaire clarté. Il suffit en effet de comparer deux passages parallèles d'Albert le Grand et de Thomas d'Aquin pour que la différence saute aux yeux : dans le même type d'ouvrage, sur la même question, en énonçant le même argument appuyé sur la même citation d'Aristote, là où Thomas, à droite, s'exprime de manière sobre, simple et limpide, à gauche Albert se montre prolixe, complexe, obscur. Là où le disciple expédie la question en quatre phrases d'une ou deux propositions, trois maximum, il faut à son maître six phrases, dont l'une se déploie sur non moins de cinquante mots rangés en neuf propositions, sans aucun souci ni de la symétrie, ni de la pédagogie. Même sur ce cas relativement simple, par rapport à ceux que nous allons étudier, il faut un peu de temps et d'attention pour être certain de hiérarchiser correctement les deux principales, l'unique subordonnée comparative et les six subordonnées relatives dont cinq se suivent en cascade.

\footnotetext{
1 Mes remerciements amicaux vont d'abord aux membres du groupe Organon : à Julie Brumberg-Chaumont, Antoni Bordoy, Michael Chase, Christophe Erismann, Marc Geoffroy, David Piché, Aurélien Robert, Philippe Vallat: la présente étude n'aurait pas été possible sans le travail collectif mené sur les textes d'Albert, pour les traduire, les annoter, les discuter, les comprendre ensemble. Merci de même à Julie Casteigt pour sa relecture vigilante et le dialogue si riche qu'elle a suscité.
} 


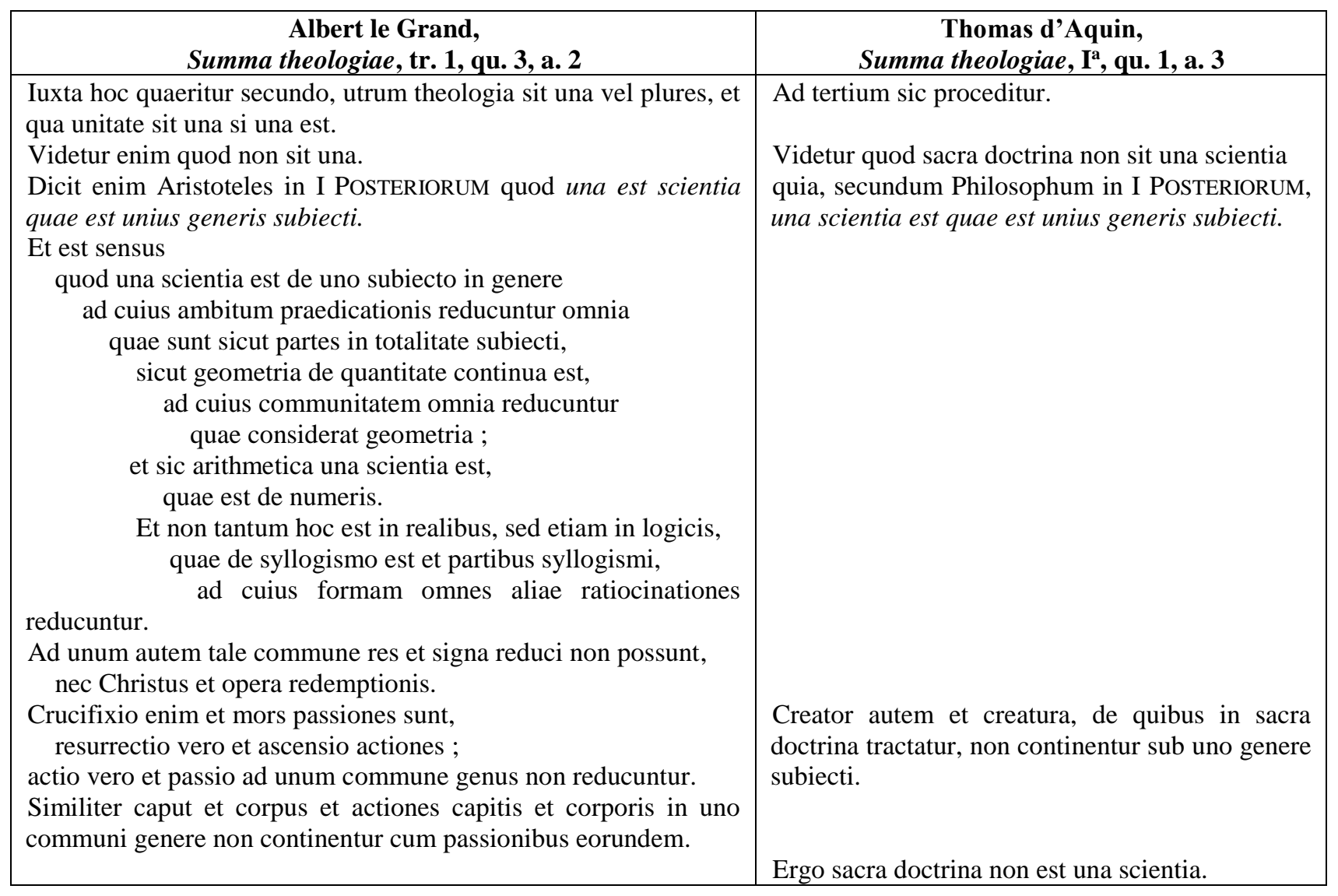

Pourquoi donc étudier le style d'un auteur qui s'en moque ? Il y a deux raisons principales à cela. La première est qu'on n'aborde pas la doctrine d'Albert sans s'affronter à ses textes. Or, qu'on le veuille ou non, ceux-ci nous parviennent hérissés de difficultés nombreuses, dont souvent on ne sait pas si leur origine est philologique, grammaticale ou conceptuelle. Devant une phrase bizarre, incorrecte ou inintelligible, qui dois-je accuser : les copistes d'avoir dégradé l'original ? L'éditeur d'avoir fait les mauvais choix ? Albert d'écrire trop vite et de ne pas se relire ? Ou Albert de trop se relire au contraire et, à chaque révision, de surcharger son ouvrage de repentirs et d'ajouts successifs, qui enflent et distendent sa phrase jusqu'à la limite de l'explosion ? Ou Albert, encore, d'être allemand et d'exprimer dans ses écrits, sinon les «brumes germaniques », du moins un sens bien transrhénan de l'Ordnung et de la Systematisierung ? Enfin, last but not least, ne dois-je pas m'accuser moi-même de ne pas l'avoir compris, de n'avoir pas su construire sa phrase ou dégager ses idées ? En pratique, le problème est que toutes ces explications s'offrent concouramment et qu'en éditant ou traduisant il faut souvent choisir entre la correction désespérée, qui brutalise le texte et banalise la pensée, et l'interprétation ingénieuse, qui respecte littéralement le premier mais entortille inutilement la seconde. À travers l'écriture d'Albert, je voudrais donc enquêter sur la genèse de ses ouvrages et l'origine de leurs anomalies.

Mais il y a une seconde raison d'étudier la phrase d'Albert. Si vraiment, selon Buffon, « le style est l'homme-même ${ }^{2} »$, si chez la même personne il existe une affinité profonde entre sa façon d'écrire et sa manière de penser, on peut espérer, en auscultant la phrase de notre auteur, découvrir quelque chose de sa méthode intellectuelle, de sa forma mentis, peut-être même de ses présupposés doctrinaux et de ses intentions de philosophe ou de théologien. En ce sens, la littérature scolastique

2 G.-L. Leclerc, comte de Buffon, Discours sur le style : prononcé à l'Académie française par M. de Buffon le jour de sa réception le 25 août 1753, J. Lecoffre, Paris 1872, p. 24. 
offre un immense chantier, vierge encore, à quiconque voudrait étudier le style des maîtres, non seulement comme une palette de couleurs posées sur une pensée déjà constituée, afin de la peindre, de la rehausser et de mieux la transmettre, mais, bien plus encore, comme le reflet de son mouvement interne et de ses tendances profondes, comme une introduction au mystère de sa naissance souterraine et de son jaillissement.

Pour ce faire, nous procèderons de la manière suivante, à partir des deux premiers traités du Super Porphyrium, étudiés dans le cadre du projet ANR/CNRS dirigé par Julie BrumbergChaumont: "L'Organon dans la translatio studiorum à l'époque d'Albert le Grand »². D'abord, nous essaierons de nous entendre sur ce qu'est la phrase d'Albert, en particulier sur ses limites. Ensuite, nous examinerons à partir de quelques exemples les principales difficultés qu'elle soulève, à la charnière bien souvent entre le textuel et le doctrinal. Enfin, nous passerons en revue les types de phrase favoris d'Albert en essayant de comprendre à quelle tendance intellectuelle ils correspondent.

\section{DELIMITER LA PHRASE D’ALBERT}

On a jusqu'ici parlé de « phrase ». Mais qu'est-ce que la phrase d'Albert et, pour commencer, où s'arrête-t-elle ? Il suffit de comparer deux éditions, Borgnet et Santos Noya par exemple, pour voir que chaque éditeur a sa façon propre de couper les phrases. Certains les aiment longues, d'autres les préfèrent brèves. Cette diversité est probablement incompressible : elle relève de l'interprétation personnelle de l'éditeur; en amont, du reste, les manuscrits eux-mêmes varient dans leur ponctuation. Sans prétendre trancher la question, on s'appuiera sur une observation simple : chez Albert, la plupart des portions de texte identifiées comme des phrases par ses éditeurs ont en commun que leur début est signalé par un mot de liaison. Ordinaire chez les auteurs latins de l'Antiquité à l'époque moderne, cet usage est quasi systématique chez Albert, comme dans l'exemple ci-dessous :

\footnotetext{
3 Nous utiliserons pour cela l'édition critique M. SANTOS NoyA (ed.), Alberti Magni Super Porphyrium De V universalibus, Aschendorff, Münster 2004 (Alberti Magni Opera omnia, t. 1, pars 1A).
} 
$<1$. $>$ Summe autem necessaria et utilis est logicalis philosophia. <2. $>$ Ex quo enim logica docet, qualiter ignotum fiat notum, patet quod in nulla philosophia aliquid notum fieri potest nisi per logicae doctrinae facultatem. <3.> Est enim, ut dicit BOETHIUs in TOPICIS, ratio disserendi, hoc est docens, qualiter de quolibet disserendum est, quae in duas, ut dicit, distribuitur partes, scilicet scientiam inveniendi, quam topicam Graeci vocaverunt, et scientiam iudicandi, quam Graeci analyticam, Latini autem resolutoriam nuncupaverunt. $<4$.> Constat autem quod sine dissertione et inquisitione non venitur de noto ad ignotum. $<5 .>$ Quae dissertio non est nisi inquisitio discutiens, qualiter notum comparatur ad ignotum et qualiter se habet ad illud ut causa vel principium, vel signum vel coniectura ad illud, vel contrario modo ut repugnans ad ipsum ; sine qua in nulla scientia aliquid sciri potest. <6.> Quoad hoc ergo quod scientia est docens disserere de omni re, non tantum est utilis in se, sed etiam necessaria est ad omne quod scire quaerimus. <7.> Propter quod dicit saepissime ARISTOTELES in diversis libris suis quod causa erroris antiquorum fuit, quia scientiam logicam non habuerunt. <8. > Docet enim logica principia, qualiter, hoc est quibus principiis et quibus argumentationibus, de quolibet scibili disserendum est. $<9$. Qui modus nisi sciatur, scibile inveniri non potest, quia is, qui quaerit, modum inveniendi scientiam scibilis illius non habet. <10.> Propter quod etiam ARISTOTELES dicit quod modus sciendi ante scientiam quamlibet discendus est, ut per illum modum scientia cuiuslibet scibilis inveniatur. <11.> Cum enim scientia habitus sit conclusionis, oportet quod concludere sciat ille qui scientiam accipit. <12.> Non autem concludere scit, qui nescit, quid concludere debeat. <13.> Et cum nihil concludi debeat nisi quaestio, oportet quod sciat, quid et qualiter quaerendum est id quod concludi intenditur. <14.> Adhuc concludere se nescit, qui nescit, ex quibus et qualibus et qua complexione concludendum est. <15.> Haec autem omnia nulla docet philosophia nisi tantum logica. <16.> Non ergo tantum utilis est et adminiculans ad omnes scientias, sed etiam necessaria. <17.> Propter quod nescientes logicam etiam id quod scire videntur nesciunt se scire, quia nesciunt, qualiter unumquodque sciri oportet et qualiter probandum vel improbandum est. Super Porphyrium, I, 3, éd. SANTOS NoYA, p. 5, 3-44.

Sur dix-sept phrases, découpées comme telles par l'éditeur, dix débutent par un adverbe de liaison (autem: 1, 4, 12, 15 ; enim : 2, 3, 8, 11 ; ergo: 6, 16; adhuc: 14), trois autres par une locution relative ayant la valeur d'un adverbe de liaison (ici la conséquence, propter quod: 7, 10, $17)$, deux autres par un pronom relatif dit lui aussi « de liaison » $(5,9)$ parce qu'il relance la phrase momentanément interrompue par un point; une dernière, enfin, débute par une conjonction de coordination (et : 13), abolissant ainsi la césure signifiée par le point. En d'autres termes, si l'on souhaite un critère simple, qui ne dépende pas des habitudes des éditeurs mais s'inscrive dans la prose même d'Albert, on pourra considérer que, sauf cas particulier, la phrase d'Albert débute régulièrement par un adverbe ou une locution adverbiale de liaison; et qu'à l'inverse les pronoms relatifs ou les conjonctions de coordination comme et, sed, nec/neque sont des artifices de l'éditeur visant précisément à distinguer plusieurs phrases, là où l'on pourrait fort bien n'en lire qu'une. Si cette phrase unique a été coupée, c'est surtout par pitié pour le lecteur moderne, qui a perdu l'habitude de phrases longues et complexes comme celles des auteurs latins, de l'Antiquité comme du moyen Âge. Toutefois il est clair que cette césure devant un relatif ou une conjonction est une option facultative, car à l'inverse il existe plusieurs cas analogues (indiqués ici par des soulignés) où l'éditeur aurait pu segmenter la phrase et ne l'a pas fait.

Quant aux mots de liaison dont Albert se sert en tête de phrase, notons qu'ils sont peu variés. Dans l'exemple retenu, on trouve autem pour le passage d'une idée à une autre, de la majeure à la mineure d'un raisonnement; enim pour une explication ou un exemple; ergo ou propter quod pour une conséquence ou une conclusion ; enfin adhuc pour passer à l'argument suivant; et c'est tout. Sans doute, sur de plus larges échantillons, on trouverait d'autres mots de liaison ; mais ceux-ci 
restent de loin les plus fréquents. Pour passer d'une phrase à une autre, Albert se sert d'un très petit nombre de mots exprimant un très petit nombre de relations logiques, principalement la causalité et la conséquence : ce qui convient bien à un discours surtout déductif, tirant de la logica utens sa force démonstrative.

\section{TYPOLOGIE DES DIFFICULTES}

À présent que nous sommes en possession d'un critère simple pour délimiter la phrase d'Albert, récapitulons les principales difficultés qu'elle offre à ses éditeurs, à ses traducteurs, ou simplement à ses lecteurs. Ces difficultés peuvent être de toute sorte : philologique, littéraire, doctrinale, mais la principale de toutes est que, face à telle ou telle phrase peu compréhensible, on ignore pour commencer à quel type de problème on a affaire et s'il faut supposer une négligence du copiste, un lapsus de l'écrivain ou une subtilité du penseur.

\section{Difficultés textuelles}

Plusieurs passages suggèrent fortement que le texte du Super Porphyrium, tel qu'il nous est parvenu, est entaché d'erreurs originelles. L'éditeur s'en est prévalu pour corriger dans certains cas le texte donné par la totalité de manuscrits examinés ${ }^{4}$. Même si, dans plusieurs cas, on pouvait conserver la leçon de ces derniers ${ }^{5}$, il n'est pas contestable que, dans plusieurs autres, le texte de l'archétype doit être corrigé, soit que tous les témoins remontent à une première copie fautive (par exemple la mise au propre de l'original d'Albert), soit que ce dernier ait lui-même introduit de menues incohérences dans son ouvrage, par exemple en le retravaillant sans se relire ensuite. Pour le philologue, de telles anomalies sont toujours passionnantes, car elles aident à comprendre les méthodes de travail de l'auteur et la genèse de sa pensée. En voici quelques exemples, concentrés dans la même portion de texte. Les passages délicats sont marqués par des gras.

En premier lieu, la proposition «et hoc iam ante nos determinavit ALFARABIUS » ne laisse pas d'étonner. Comme nous l'a fait remarquer Marc Geoffroy par oral, la référence est vague et laisse insatisfait, à tel point que l'éditeur a supposé qu'Albert pourrait se référer à Gundisalvi plutôt qu'à al-Farabi. Or si le renvoi intrigue à cette place, en revanche il s'adapte parfaitement à ce qui suit, puisque, aussitôt après, le texte d'Albert est clairement démarqué d'al-Farabi. Pourtant, le tamen de la phrase suivante pose une frontière claire entre les deux développements : la référence à al-Farabi devait donc se rapporter à ce qui précède et non à ce qui suit. Que s'est-il passé ? Une hypothèse vraisemblable est qu'Albert, révisant son texte, ajouta dans la marge une référence au philosophe arabe et qu'au moment de la mise au propre celle-ci fut insérée à la mauvaise place, juste un peu trop haut.

\footnotetext{
Hae igitur sunt partes, quae generaliter habent docere modum accipiendi scientiam de quolibet scibili incomplexo vel complexo,

et hoc iam ante nos determinavit AlFARABIUS.

Super Porphyrium, I, 7, éd. SANTOS NOYA, p. 15, 42-45.
}

\footnotetext{
4 <convenienti> au lieu de consequenti, Super Porphyrium, II, 7, éd. SANTos NoyA, p. 36, 62-63; même chose, ibid., p. 36, 64.

Par exemple, essentia a été méthodiquement remplacé par existentia contre le témoignage des manuscrits, Super Porphyrium, I, 3, éd. Santos NoyA, p. 4, lignes 36, 39 et 43, alors que plus loin Albert lui-même pose les deux notion d'existentia et d'essentia comme interchangeables: «Et secundum hoc intelligitur quod existentiae rerum sive essentiae, sicut lumina agentis accepta, incorruptibilia sunt et ingenerabilia», ibid., II, 3, p. 26, 2-4.
} 
La phrase suivante pose un problème légèrement différent. Parlant de la méthode par laquelle la logique procède du connu vers l'inconnu, Albert ajoute - d'après al-Farabi cette fois - que la manière de procéder de la logique (Hic tamen modus) se diversifie (variatur) «selon la matière dans laquelle on la place » (secundum materiam in qua ponitur), et « selon la diversité de la matière en laquelle on cherche la science » (secundum diversitatem materiae in qua quaeritur scientia). À l'évidence, ces deux compléments de variatur sont redondants, la seule différence étant que le second est un peu plus précis que le premier. Il est donc tentant de voir dans cette répétition les vestiges d'un travail de récriture. Mécontent de sa première formulation trop vague, Albert a pu rédiger la seconde dans l'interligne. Puis, lors de la mise au propre, son secrétaire se sera trompé : prenant pour un ajout ce qui était une correction, il aura conservé les deux leçons, au lieu de remplacer l'ancienne par la nouvelle.

\author{
Hic tamen modus \\ secundum materiam in qua ponitur, \\ variatur \\ secundum diversitatem materiae in qua quaeritur scientia. \\ Super Porphyrium, I, 7, éd. SANTOS NOYA, p. 15, 45-47.
}

Ensuite s'ouvre une longue phrase, sur laquelle nous reviendrons à un autre point de vue. Pour le moment, observons seulement qu'elle contient un solécisme répété. Énumérant les parties de la logique: grammaire, poétique, rhétorique, etc., Albert écrit un singulier pour la première (grammatica), un pluriel neutre pour les deuxième et troisième (poeticis, rhetoricis), mais emploie dans les trois cas un verbe au singulier: (aliter est in grammatica, quae... attendit; aliter est in poeticis, quae... intendit; et aliter est in rhetoricis, quae... docet). Deux explications principales peuvent être envisagées. Ou bien Albert travaillait par strates textuelles. D'abord il s'est contenté d'énumérer les parties de la logique : «il en va autrement en grammaire, autrement en poétique, autrement en rhétorique », puis, dans un second temps, a précisé le contenu de chaque discipline, oubliant alors qu'il avait oscillé entre le singulier et le pluriel. Ou bien, Albert écrivait vite, était peu lisible ou employait des abréviations trop sévères, en sorte que certaines, incorrectement résolues, ont introduit un pluriel là où il y avait un singulier (poetica, rhetorica), ou posé un singulier là où il y avait un pluriel (intendunt, docent).

Une autre anomalie dans les environs suggère que le passage tout entier conserve les cicatrices d'une hâte ou d'une corruption originelle. On lit en effet : et ideo fabula et recitatione, là où il faut probablement lire et ideo in fabula et recitatione, analogue à l'in oratione qu'Albert écrit juste après. Là aussi, on peut invoquer une confusion entre ideo et in, soit que le secrétaire ait lu un mot à la place de l'autre, soit que l'ajout entre les lignes d'un ideo ait été interprété par erreur comme une correction au lieu d'un ajout : ce serait alors une faute inverse de celle qu'on a examinée à l'instant. Dans tous les cas, cette accumulation d'anomalies sur un même passage suggère fortement qu'en cet endroit l'archétype offrait un texte incorrect, soit parce qu'un premier état du texte peu lisible a facilité des erreurs de copie, soit parce que l'auteur lui-même, revenant sur ce paragraphe, l'a surchargé d'ajouts qui en ont altéré la cohérence grammaticale. 


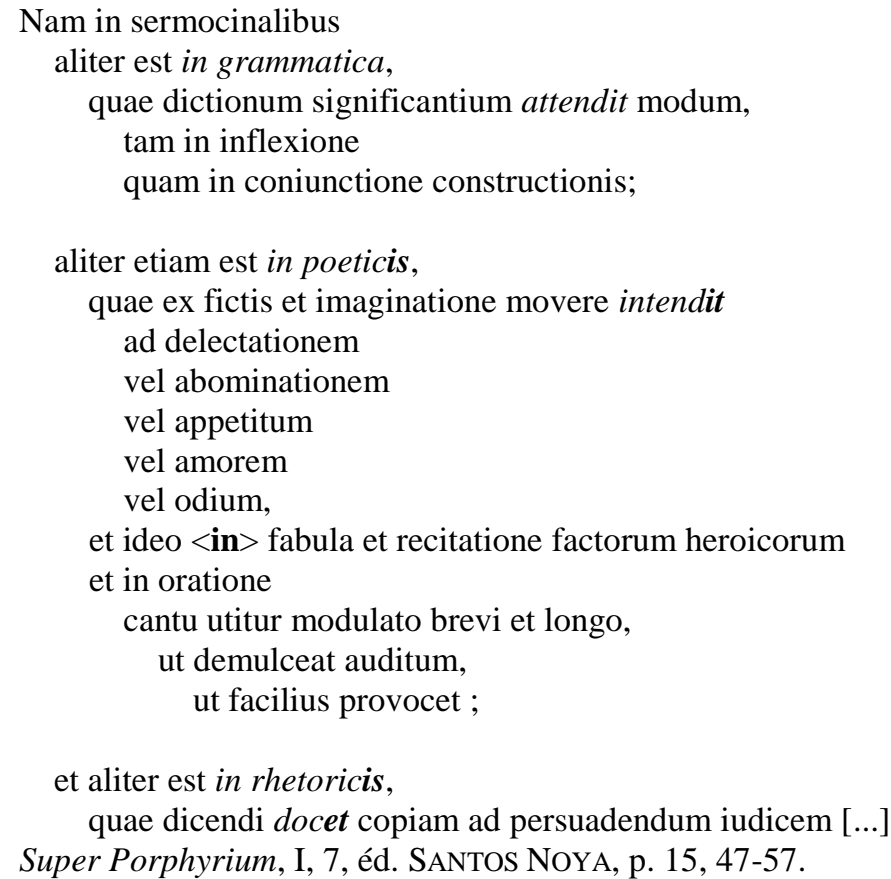

\section{Lapsus de l'auteur}

Comme Homère, Albert a pu sommeiller quelquefois. Certains passages ne peuvent s'expliquer que par un lapsus de l'auteur, notamment lorsqu'il se contredit ouvertement. Énumérant les cinq préceptes de la définition, Albert mentionne en troisième lieu la nécessité de présenter ses éléments en bon ordre, en sorte que le déterminant vienne toujours après le déterminé, comme l'acte après la puissance. L'exemple qu'il donne, à propos de l'homme, présente ces cinq éléments : animal, sensible, rationnel, mortel, apte à se mouvoir volontairement. Hormis l'ajout de « sensible », cela correspond bien à la définition qu'il a donnée plus haut, à un détail près ${ }^{6}$ : au lieu qu'un processus de précision croissante juxtapose les déterminations dans cet ordre : « animal », « rationnel », puis «sensible », et «apte à se mouvoir à volonté », on lit dans la phrase en question que, de même qu' « animal » est déterminé par « sensible » et «sensible» par « rationnel », de même «mortel» est déterminé par « rationnel » et « apte à se mouvoir volontairement » par « mortel ».

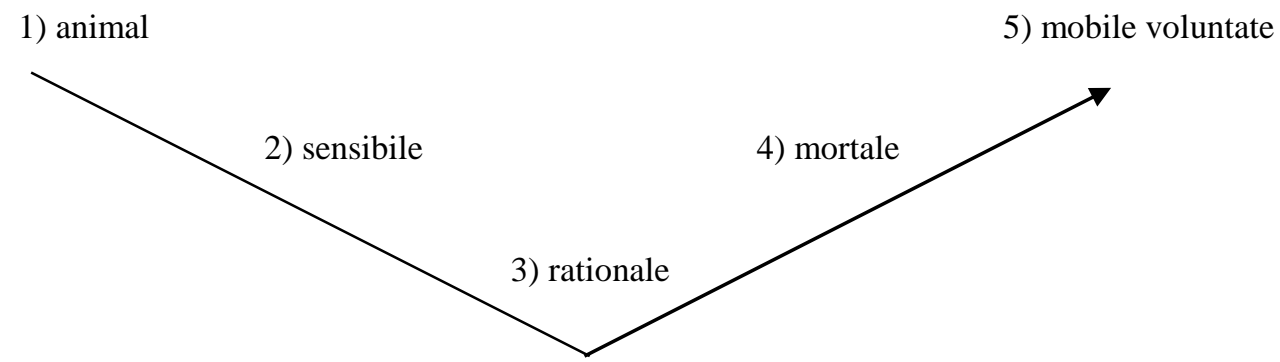

\footnotetext{
Cf. « [...] sicut cum dicimus 'animal rationale, mortale, voluntarie mobile' diffinitionem esse hominis. 'Animal' enim, quod in se claudit corpus animatum sensibile et substantiam, prima potentia est, in qua incohatum est esse hominis. Haec autem incohatio per 'rationale' determinata est essentialiter ad actum rationis. Per 'mortale' autem ad speciem est determinatum [...]. Per hoc autem quod 'mobile voluntate' dicitur, separatur ab aliis, quae acta a natura et phantasia sunt ad motum », ibid., p. 11, 24 - 12, 3.
} 
$\mathrm{Au}$ lieu donc d'une série de termes allant linéairement du moins au plus déterminé, la série bifurque à «rationnel », qui détermine à la fois « sensible » et «mortel », au contraire de ce qui avait été énoncé plus haut. C'est sûrement par erreur qu'Albert a inversé par l'ordre des mots «mortale ad rationale et sicut mobile voluntate ad mortale ». Il faut donc comprendre autre chose que ce qui est écrit et lire, moyennant deux permutations : «et sicut animal ad sensibile et sicut sensibile ad rationale et sicut rationale ad mortale et sicut mortale ad mobile voluntate ».

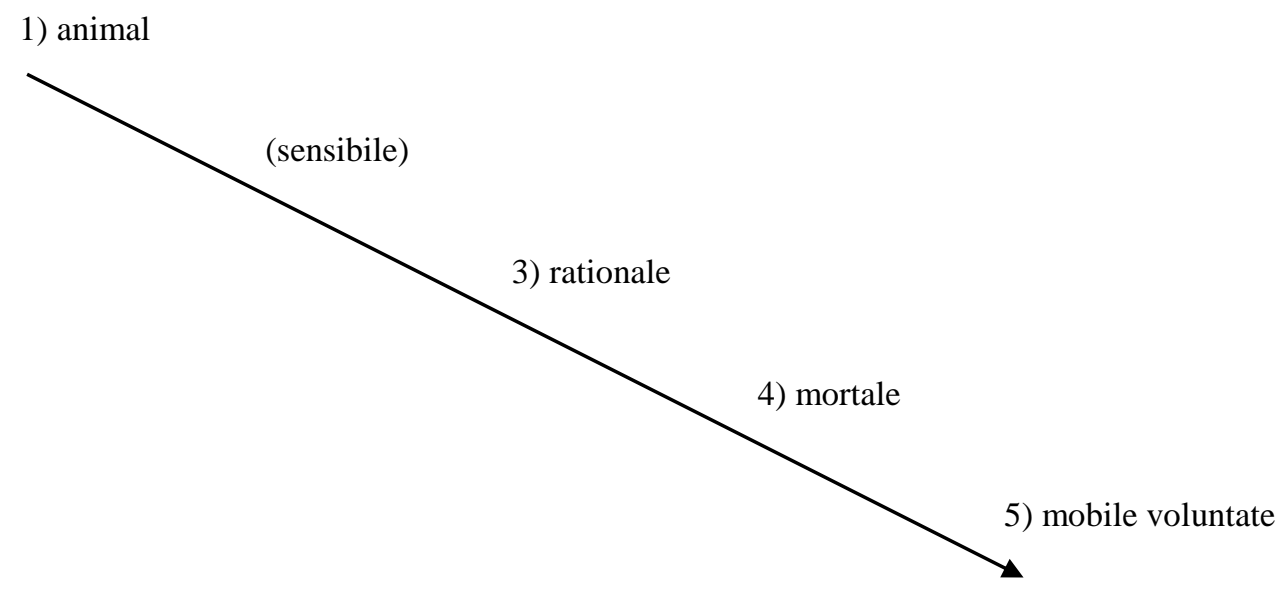

Tertium autem

quod prius in diffinitione se habeat ad sequens,

sicut potentia propinqua ad actum

et sicut determinatum ad determinans

et sicut distinctum ad distinguens

sicut animal ad sensibile

et sicut sensibile ad rationale

et sicut mortale ad rationale

et sicut mobile voluntate ad mortale

ita quod in uno motu generationis semper posterius procedat ex antecedente.

Super Porphyrium, I, 7, éd. SANTOS NOYA, p. 12, 10-16.

\section{Difficultés doctrinales}

Le contrecoup le plus dangereux de ces erreurs d'archétype et de ces lapsus d'auteur, c'est qu'une fois mis en évidence ils encouragent le lecteur à en chercher là où, peut-être, il n'y en a pas, spécialement dans les passages obscurs d'Albert, qui ne sont pas rares. Presque chaque page en contient : je n'en citerai qu'un, sans être totalement sûr d'avoir saisi son interprétation correcte. Exposant la thèse réaliste selon laquelle les universaux existent dans les intellections «nues et pures "), Albert commence par présenter une objection tirée d'Aristote et de Boèce : l'universel, disent-ils, ne peut pas être séparé dans la nature, puisque tout ce qui est séparé dans la nature est unum numero. Or un universel comme le genre ou l'espèce n'est pas unum numero, mais unum in multis et de multis. Puis il donne la parole à la réfutation réaliste de cette objection, appuyée sur deux arguments. Le premier consiste à supposer qu'Aristote et Boèce parlent seulement des réalités « ultimes », totalement accomplies en acte dans la nature, ce qui exclut les universaux. Le second envisage l'hypothèse où Aristote et Boèce incluraient bien les universaux dans leur objection, mais même alors « on comprend qu' unum numero se dit à cause du nombre de l'être ultime » (a numero ultimi esse), « et non à cause du nombre de l'essence »(a numero essentiae). 
À première vue, ce second argument est tout à fait obscur et paradoxal : on s'attend à ce qu' « un numériquement » se dise de l'essence, qui est une, plutôt que de l'être des réalités concrètes, qui sont plurielles. La contradiction disparaît toutefois si l'on admet qu'Albert joue en quelque sorte sur les mots et prend au sens fort l'expression unum numero. Puisqu'à proprement parler « un » n'est pas un nombre, l'expression unum numero, problématique en elle-même, devient comme la clef du problème des universaux, pour peu qu'on ne l'entende pas simplement au sens $d$ ' « un numériquement », mais que l'on comprenne : « un par le nombre », « un dans le nombre », presque « un à partir $d u$ nombre et en dépit $d u$ nombre ». Entendu de cette façon, qu'y a-t-il de plus unum numero que l'universel, puisque d'un côté il est «un» par l'essence, et que de l'autre il est «nombreux » par les êtres ultimes qui la réalisent? Le texte d'Albert s'éclaire alors : unum numero se dit donc bien «à partir du nombre (a numero) de l'être ultime, et non à partir du nombre de l'essence.

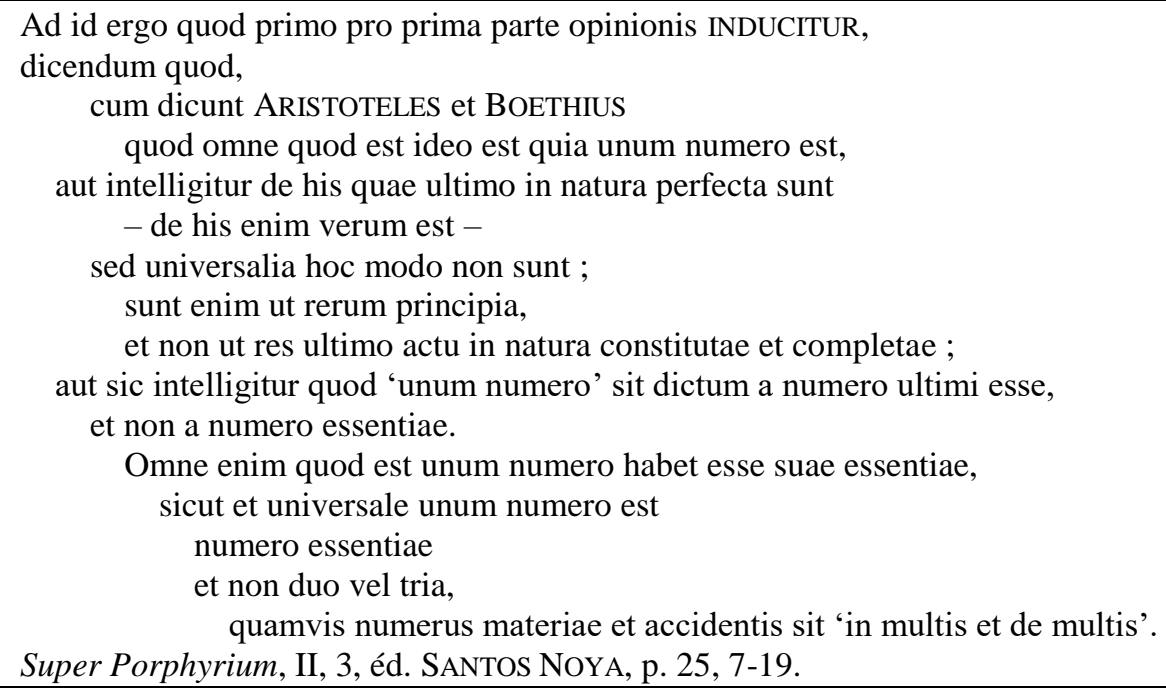

\section{Difficultés de construction}

En plus des difficultés de toute sorte qu'on vient d'étudier, mais se confondant souvent avec elles, il en existe une nouvelle sorte : ce sont les difficultés syntaxiques soulevée par la phrase d'Albert, dès lors qu'elle se développe et atteint une certaine longueur et complexité. C'est ce que nous allons maintenant étudier, en esquissant une typologie de ses phrases longues.

\section{TYPOLOGIE DES PHRASES D’ALBERT LE GRAND}

\section{Phrase ornée}

S'il est un type de phrase longue que je n'ai pas trouvé dans le Super Porphyrium, même à son début, c'est la phrase d'apparat, périodique, rimée et rythmée, qu'on lit à la même époque chez divers maîtres comme Bonaventure, y compris dans des textes scolaires comme son commentaire aux Sentences de Pierre Lombard: 


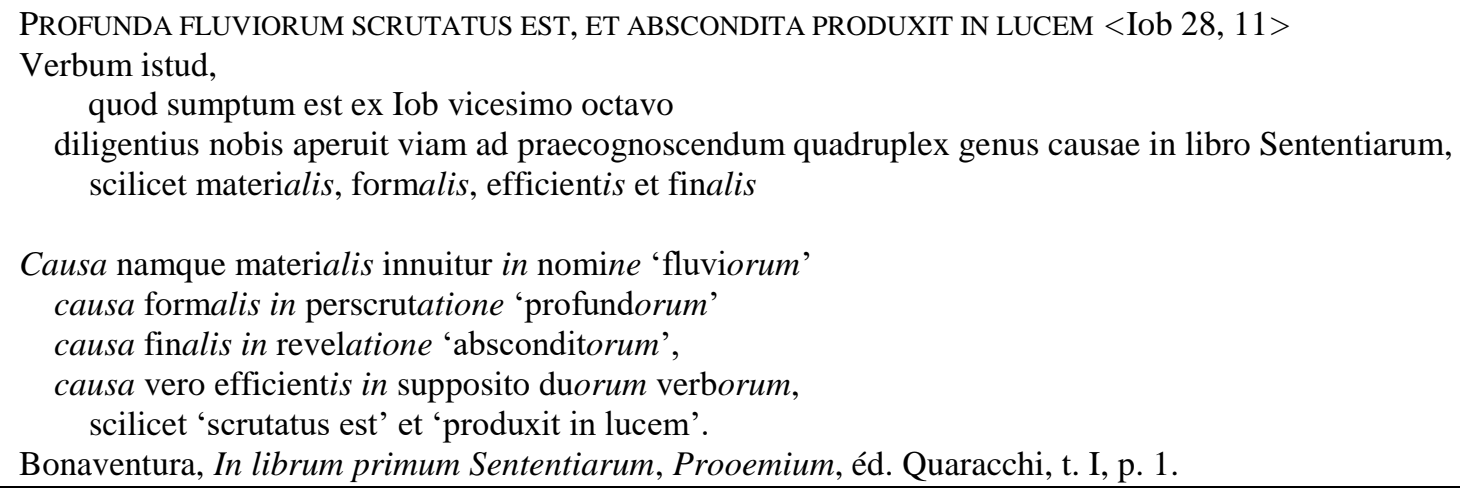

Décortiquant un verset de Job, Bonaventure parvient non seulement à y retrouver les quatre causes d'Aristote, mais encore à exprimer son commentaire de ce verset dans une prose d'art où, dans la seconde phrase, on note les quatre premiers membres unis par le nombre (entre 17 et 21 syllabes), le rythme (deux cursus veloces, puis deux cursus plani) et la rime : principale en -orum, secondaire en -alis, -atione/ne, -tur, sans compter l'anaphore du mot causa.

D'une façon générale, la phrase d'Albert est plutôt à l'opposé de la phrase bonaventurienne : sans rime, irrégulière, dissymétrique. Il y a cependant quelques exceptions partielles, comme dans le cas suivant où notre auteur définit les deux parties de la logique, selon qu'elles traitent des expressions « incomplexes » et « complexes ». Entre ces deux parties, il esquisse en effet un certain parallèle: la définition et le syllogisme, qu'étudient ces deux sciences, sont dits leurs « instruments »; parmi leurs sous-parties, sont énumérées de part et d'autre : « ce qui environne » la définition ou le syllogisme, et pour finir «ce qui imite » la définition ou le syllogisme. Mais la symétrie ainsi esquissée tourne court : du reste, la science de l'incomplexe comporte cinq parties, celle du complexe en a six. En outre le parallèle n'est appuyé par aucun effet syllistique, de rime ou de rythme, hormis ceux que suscitent naturellement de fréquentes répétitions. Bref le lecteur habitué à Bonaventure ou même à Thomas reste sur sa faim. Pourtant, il manquait peu de chose pour transformer la phrase d'Albert en une belle période, aussi agréable à entendre et à lire que facile à comprendre et à mémoriser : Albert n'en a pas eu l'idée, ou l'envie, ou le temps. 
Sicut autem logicus,

docens quaerere scientiam incomplexi,

docet instrumentum,

quo accipiatur notitia illius,

scilicet diffinitionem

et ea quae ad diffinitionem faciunt

et quae diffinitionem circumstant

et quae diffinitionem perficiunt

et ea quae diffinitionem imitantur,

sic

docens accipere scientiam complexi

docet syllogismum,

qui est illius proprium instrumentum,

et docet

alias species argumentationum

et principia syllogismi

et ea quae circumstant ipsum,

et principia ipsius et partes et materiam in qua potest poni forma syllogismi

et aliarum argumentationum forma quae syllogismum imitantur.

Et ideo ea,

de quibus habet tractare logicus,

secundum ista dividuntur et multiplicantur.

Super Porphyrium, I, 7, éd. SANTOS NoYA, p. 14, 42-54.

\section{Phrase germinale}

Si notre auteur n'use pas du style périodique, comment s'y prend-il pour obtenir des phrases aussi longues? De plusieurs manières. L'une d'elle consiste à partir d'une phrase assez simple au départ, mais sur laquelle des développements secondaires se greffent de façon irrégulière et imprévisible. On dirait de grands arbres, dont les branches maîtresses sont bien réparties, mais se ramifient et se démultiplient ensuite de manière très inégale, déséquilibrant la silhouette de l'ensemble. Telle la phrase suivante sur les trois états de l'universel, ante rem, in re et post rem. Au départ, la structure générale est clairement marquée :

Et hoc est quod dixerunt antiqui triplices esse formas :

ante rem scilicet [...],

et in re sive cum re ipsa [...],

sunt etiam formae post rem [...],

Elle est même soulignée par une triade de relatives, à peu près analogues et proportionnées :

Et hoc est quod dixerunt ANTIQUI triplices esse formas :

ante rem scilicet,

quae sunt formae secundum se acceptae [...],

et in re sive cum re ipsa,

quae sunt formae existentes in ipsis [...],

sunt etiam formae post rem,

quae sunt formae per abstractionem intellectus ab individuantibus separata [...]

Toutefois, l'un des membres - ici le second - absorbe la sève commune : 
Et hoc est quod dixerunt ANTIQUI triplices esse formas :

ante rem scilicet,

quae sunt formae secundum se acceptae,

principia rerum existentes ;

et in re sive cum re ipsa,

quae sunt formae existentes in ipsis,

dantes eis nomen et rationem

per id quod sunt aptae esse in multis et universales,

non tamen secundum quod sunt in istis [...]

sunt etiam formae post rem,

quae sunt formae per abstractionem intellectus ab individuantibus separata

et 'in quibus intellectus agit universalitatem'.

Il se met à croître, jusqu'à former à lui seul plus de la moitié de la phrase complète :

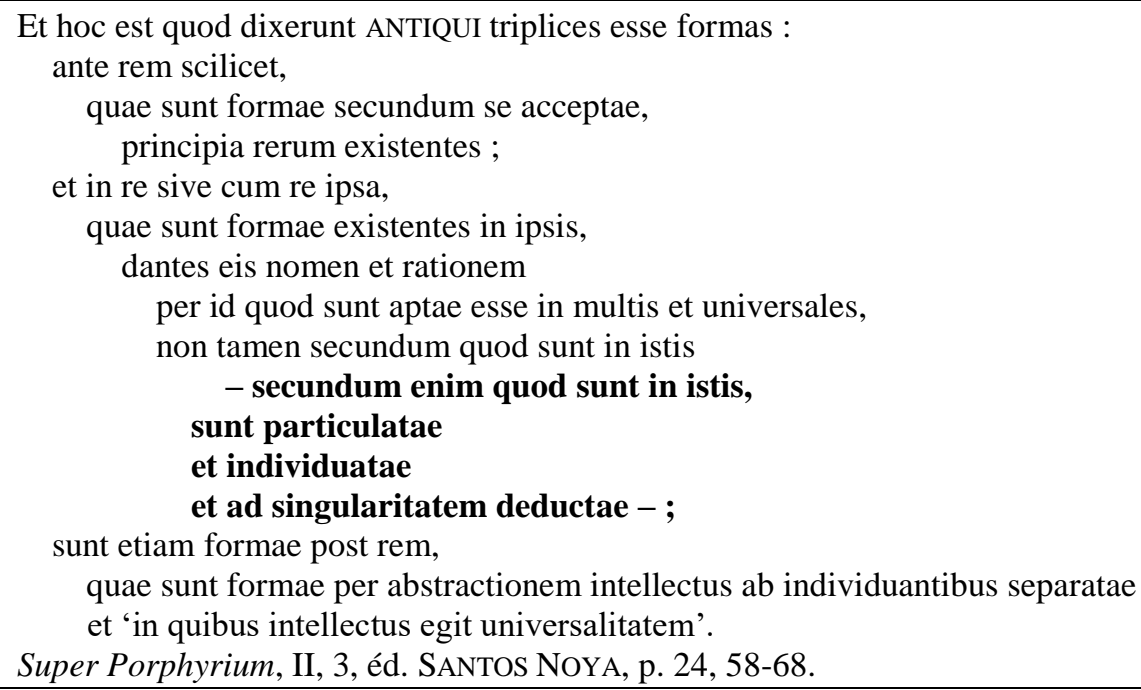

Et hoc est quod dixerunt ANTIQUI triplices esse formas :

ante rem scilicet,

quae sunt formae secundum se acceptae,

principia rerum existentes ;

et in re sive cum re ipsa,

quae sunt formae existentes in ipsis,

dantes eis nomen et rationem

per id quod sunt aptae esse in multis et universales,

non tamen secundum quod sunt in istis

- secundum enim quod sunt in istis,

sunt particulatae

et individuatae

et ad singularitatem deductae - ;

sunt etiam formae post rem,

quae sunt formae per abstractionem intellectus ab individuantibus separatae

et 'in quibus intellectus egit universalitatem'.

Super Porphyrium, II, 3, éd. SANTOS NoYA, p. 24, 58-68.

\section{Phrase arborescente}

Une autre manière, tout aussi efficace, d'obtenir une phrase longue consiste à y renfermer la description d'un objet complexe, par exemple une science avec ses divisions, en sorte que les articulations principales puis secondaires de la phrase correspondent aux ramifications principales puis secondaires de cette science. On obtient ainsi une phrase arborescente, dont la construction grammaticale imite la structure réelle de ce qu'elle exprime. Souvent alors, tandis qu'Albert se contente au début d'annoncer les parties principales, en pratique il n'en décrit pas moins toutes leurs subdivisions, comme si sa pente intellectuelle l'entraînait inexorablement à démultiplier de façon de plus en plus fine le contenu de la chose étudiée. Ainsi, voulant dire que la logique se compose de deux parties, selon qu'elle porte sur les expressions incomplexes ou complexes, il poursuit en donnant le détail des cinq branches en lesquelles se distribue la seconde partie : 
Istae igitur sunt duae partes logicae :

una quidem,

ut dentur principia,

per quae sciatur diffinitio rei et quiditas,

ita quod per principia illa doceatur,

quae sit vera rei diffinitio et quae non,

et quae videatur esse et non sit ;

alia vero,

ut doceantur principia,

qualiter per argumentationem probetur enuntiationis veritas vel falsitas,

et ut doceatur illius argumentationis forma quantum ad figuram et modum et coniugationem sive complexionem,

et ut doceatur eiusdem argumentationis materia,

et ut doceatur quae videatur esse argumentatio et non sit,

eo quod apparentiam aliquam quidem habet, sed veram existentiam non habet,

et ut doceantur cautelae,

quibus opponens vel respondens

per aliquam occasionem

impediatur vel promoveatur a conatu veritatis,

vel ad hoc quod perspecte videat veritatem et non divertat ab ipsa.

Super Porphyrium, I, 5, éd. SANTOS NOYA, p. 8, 38-53.

\section{Phrase inventaire}

Plus loin, Albert récidive et donne suivant al-Farabi une division plus vaste encore de la logique, selon les domaines du savoir où elle a l'occasion de s'exercer : sciences du langage comme la grammaire, la poésie, la rhétorique judiciaire, la rhétorique morale ; sciences de la réalité classées selon leur degré de certitude en probables, nécessaires et conjecturales. La matière, la méthode et la fin de chacune de ces sciences sont ensuite détaillées par le menu au moyen de subordonnées relatives, participiales ou finales, le tout formant une phrase unique, dont l'arborescence se déploie jusqu'au quatrième niveau. Encore l'énumération n'est-elle pas close, puisqu'elle se termine par un et aliis huiusmodi scientiis un peu énigmatique, dont on ne voit pas bien s'il ouvre la porte à d'autres sciences conjecturales, à d'autres sciences réelles, ou à d'autres sciences quelconques. Cet « etc. » au bas d'une division des sciences n'est pas rare chez Albert ; au contraire c'est même une attitude assez fréquente chez lui. Après tout, puisque les sources arabes l'ont déjà conduit à élargir l'Organon, qui sait si des traductions nouvelles n'inviteront pas les Latins à étendre encore leur corpus des textes et des savoirs? En se gardant de clore trop vite le canon des sciences, Albert se montre un témoin prudent de son époque, temps de changements accélérés dans l'organisation des connaissances. Comme les archivistes allemands ouvrent des dossiers vides en prévision des documents futurs, ou éventuels, qui doivent un jour y trouver place, de même Albert, passionné des corpus et des arborescences, cherche indéfiniment à produire les classements non seulement les plus fins et les mieux articulés, mais aussi les plus complets et les plus ouverts : rien ne doit échapper à son penchant taxinomique. De là une phrase tentaculaire, parce qu'elle cherche à tout embrasser ; une phrase inventaire, une phrase encyclopédique. 
Nam in sermocinalibus

aliter est in grammatica,

quae dictionum significantium attendit modum,

tam in inflexione

quam in coniunctione constructionis;

aliter etiam est in poeticis,

quae ex fictis et imaginatione movere intendit

ad delectationem

vel abominationem

vel appetitum

vel amorem

vel odium,

et ideo <in> fabula et recitatione factorum heroicorum

et in oratione

cantu utitur modulato brevi et longo,

ut demulceat auditum,

ut facilius provocet ;

et aliter est in rhetoricis,

quae dicendi docet copiam ad persuadendum iudicem,

ut provocet ad vindictam

vel praemiationem eius

de quo persuadet,

sumens locos rhetoricos

tam in persuadendo

quam etiam in conquestione et accusatione,

et sumit etiam colores orationis,

ut lepida sit oratio,

et ideo audientibus magis accepta propter culturam sermonis ;

aliter etiam est in laudabilibus et ethicis,

in quibus laudis vel vituperationis demonstratio acceptabile facit quod proponitur, plus quam virtus persuasionis ;

et in realibus scientiis

aliter est in probabilibus,

et aliter in necessariis et stantibus,

et aliter est in coniecturalibus

quae sunt scientiae divinationum,

sicut in physiognomia

et secunda parte astronomiae

et in geomantia,

nigromantia

et aliis huiusmodi scientiis.

Super Porphyrium, I, 7, éd. SANTOS NoyA, p. 15, 47-70.

\section{Phrase interpolée, spécialement en exégèse}

Un autre type de phrase longue est la phrase interpolée, particulièrement fréquente en exégèse. J'entends par là une phrase dans laquelle un discours principal est comme entrelardé d'incises explicatives. Cette façon de faire suppose que le lecteur ait sous les yeux le texte commenté, ou le connaisse par cœur. Moyennant quoi, ce mode d'écriture permet à notre auteur de coller à la lettre du texte, qu'il insère dans sa phrase, tout en ajoutant des élucidations, par exemple des synonymes introduits par «vel », «sive », « id est» ou «scilicet»: ici «speculatione sive consideratione »; ou bien en insérant des précisions supplémentaires : par exemple le nombre «cinq » à propos de «ces choses» dont parle Porphyre, ce qui permet de comprendre immédiatement qu'il s'agit toujours des universaux; ou bien des commentaires plus ou moins longs, sous la forme de 
propositions incises (de « modus enim » à « sufficit»), de propositions circonstancielles (de « Cum igitur » à «sunt »). Dans cette phrase interpolée, le texte commenté (ci-dessous en petites capitales) occupe normalement la position principale, et les interventions d'Albert s'accrochent à lui comme des gloses périphériques :

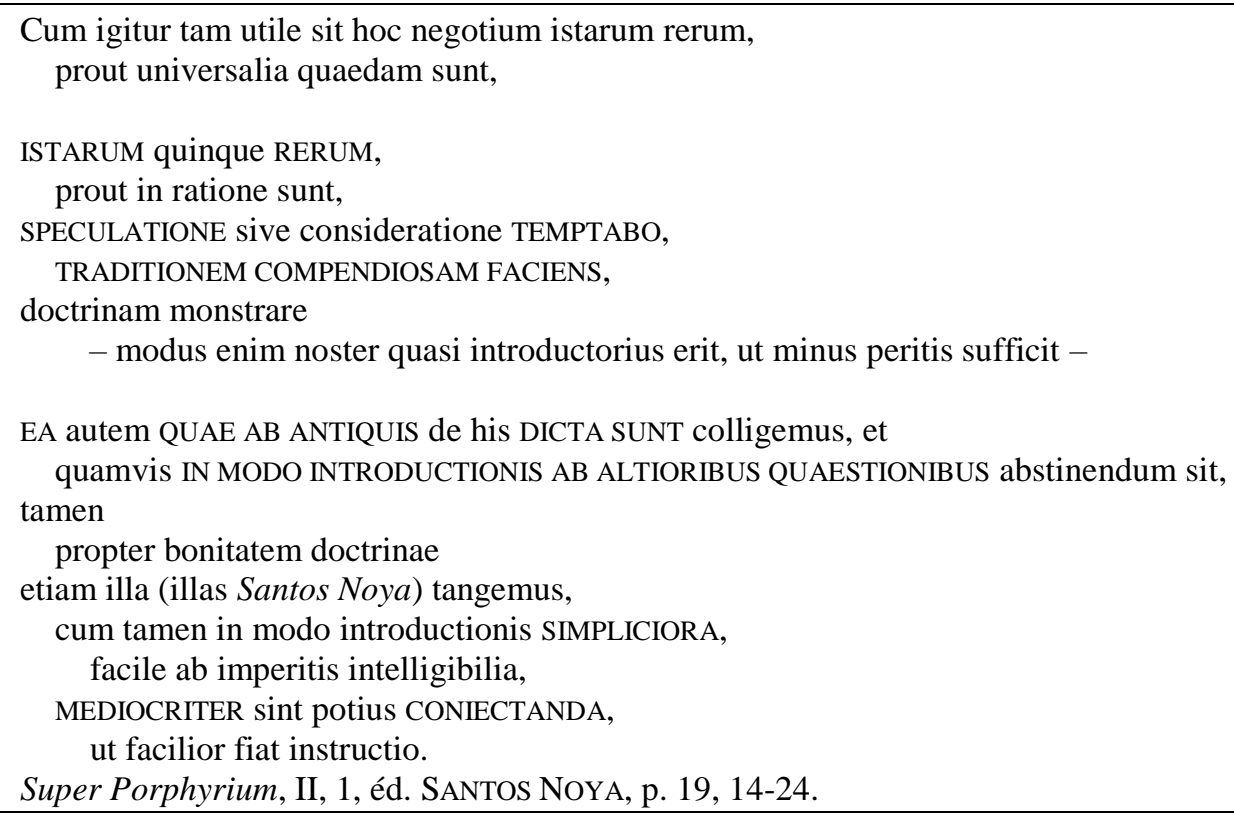

Il y a cependant des exceptions, comme ici, dans la seconde partie de la phrase, où le texte de Porphyre se trouve pour l'essentiel rejeté dans deux subordonnées concessives ( quamvis in modo introductionis ab altioribus quaestionibus abstinendum sit»; "cum tamen in modo introductionis simpliciora, facile ab imperitis intelligibilia, mediocriter sint potius coniectanda », tandis que l'interprétation d'Albert occupe la principale principale, juste entre les deux : «tamen propter bonitatem doctrinae etiam illa tangemus $\gg$. On ne saurait mieux s'y prendre pour contredire Porphyre sous le couvers de son autorité. Ce que le texte déclare ici, c'est en effet que même s'il vaut mieux s'abstenir des questions trop hautes (dixit Porphyre), il est préférable néanmoins d'en parler tout de même (dixit Albert).

\section{Phrase « en cascade »}

Un autre type de phrase longue est ce qu'on pourrait appeler la phrase en cascade. D'abord, la phrase commence comme si elle devait s'achever assez vite ; mais, peu à peu, la voici rallongée, une première fois, puis une deuxième, puis une troisième... et ainsi de suite jusqu'à une sixième fois, grâce à un procédé très simple : chaque nouveau membre est une proposition relative, rattaché au dernier mot important d'un membre précédent. Cette phrase longue est en réalité une phrase rallongée.

Similiter igitur, ut DICUNT, alicuius philosophiae erit intentio comprehendere veritatem eius, quod in nobis est secundum rationem, qua nititur comprehendere veritatem eius

quod rationis ductu via est in omnem cognitionem omnium eorum quorum cognitio fit in nobis per ea quae cognita sunt apud nos, ex quibus nos proficimus ad scientiam incognitorum.

Erit igitur de intentione philosophiae etiam logica scientia sive rationalis. Super Porphyrium, I, 2, éd. SANTOS NOYA, p. 3, 38-46. 
En l'occurrence, le lecteur n'échappe pas à une impression de tâtonnement et de piétinement. En effet, tout ce qu'Albert signifie dans cette phrase à tiroirs successifs, tiendrait en une proposition unique : «Le but de la philosophie, c'est de comprendre, en nous et selon la raison, la vérité de l'inconnu au moyen du connu. » Pour exprimer cette idée, on le voit se répéter en employant plusieurs fois les mêmes notions ou expressions : « ...comprehendere veritatem eius quod» (2 fois) «... in nobis» (2 fois) «... secundum rationem ou rationis ductu...» (2 fois) « cognitio» ou « cognita» ( 3 fois). Bref, la lourdeur et la complexité grammaticale de la phrase semblent ici superflues, sauf à supposer qu'elles imitent ce dont il est question, c'est-à-dire ce progrès discursif, réflexif et difficultueux par lequel la raison avance pas à pas du connu à l'inconnu en faisant retour sur elle-même. Un tel souci de mimétisme stylistique est peu probable; mieux vaut admettre qu'Albert, en commençant sa phrase, ne sait pas encore comment il l'achèvera. Il est alors comme un ingénieur qui a construit sa première tête de pont sur une rive, et lance l'ouvrage au-dessus de l'eau sans savoir où il placera les arches intermédiaires, mais prévoit, ou parie, qu'il trouvera bien où les faire reposer. Ces arches, construites à la hâte, ce sont nos six relatives, qu'Albert élève au fur et à mesure pour soutenir un pont qui n'attend pas, mais avance comme il peut. Albert n'est pas un auteur méditatif. Il n'est pas, comme son disciple, un «bœuf muet » qui réfléchit longuement avant de remplir l'univers de ses mugissements; c'est au contraire un auteur qui écrit vite, qui pense en écrivant, peut-être même qui écrit pour penser. Albert s'est-il relu ? S'il l'a fait, il paraît s'être plus soucié d'ajouter de la matière supplémentaire que d'élaguer ses propos antérieurs. Ses retouches vont dans le sens de la ramification et de la complexification croissantes, non dans celui de la simplicité.

\section{Phrase « question - réponse »}

Un autre type de phrase complexe renforme une sorte de dialogue. Dans la première partie, Albert présente une objection; dans la seconde, il y répond. On peut alors se demander pourquoi notre auteur n'a pas rédigé deux phrases, comme il aurait été plus clair, l'une présentant l'objection, l'autre sa réfutation. La réponse la plus probable est que le dominicain, en écrivant, ne sépare pas sa démarche en deux moments, qui alternent le faux et le vrai ; mais, au long de sa quête, il a l'esprit tiraillé entre les diverses thèses qu'il confronte. S'il finit, bien sûr, par en épouser une et une seule, ce n'est qu'à condition d'avoir épuisé tout ce qu'il y a de solide et de valide dans toutes les thèses adverses. La conclusion d'Albert n'écarte pas une antithèse, elle la surmonte après s'en être nourrie le plus longtemps possible. Son style en témoigne, le rêve philosophique d'Albert, c'est de tout absorber, de tout accorder, de créer entre Athènes, Rome, Alexandrie et Bagdad un consensus auquel - nous le savons aujourd'hui - les intéressés n'auraient peut-être pas songé, mais qu'importe : cette ardeur à tout intégrer témoigne d'une confiance peu commune en une raison universelle.

Si autem sic obiciat :

'Quod est in individuo est in eo per modum individui ;

individuum autem est hoc aliquid ;

ergo quod est in individuo, est singulare et hoc aliquid',

non valet argumentum.

Super Porphyrium, II, 3, éd. SANTOS NoyA, p. 25, 42-45. 
Si forte aliquis dicat

quod illa ratio non probat

quod universale habeat esse discretum,

sed probat

quod singulari destructo remanet esse universalis secundum aliquem modum,

hoc videtur contra ARISTOTELEM,

qui,

ponens quod universale esse habeat in singulari,

dicit quod destructis primis impossibile sit aliquid aliorum manere.

Super Porphyrium, II, 5, éd. SANTOS NOYA, p. 31, 9-15.

Si autem aliquis dicat quod,

ut dicit ARISTOTELES,

'moventibus nobis moventur ea quae in nobis sunt',

et quod hoc intelligitur de motu corruptionis,

et sic corruptis primis impossibile est universale remanere,

dicendum quod hoc omnino est verum

quod dicit Aristoteles,

et ita intelligit sicut dictum est,

et verum est quod universale corrumpitur in eis quae corrumpuntur,

sed corrumpitur secundum quod est in eis.

Super Porphyrium, II, 5, éd. SANTOS NOYA, p. 33, 37-44.

Il peut arriver qu'entre l'objection et sa réponse s'intercale une incise, si longue qu'il ne reste plus qu'à clore la première phrase pour en créer une nouvelle. Le résultat est alors que la première contenant l'objection reste en suspens. Dans l'exemple suivant, la subordonnée « Si dicatur... » demeure sans principale, sauf à supposer que 1' "Ad haec autem non est difficile respondere », en dépit de l'adverbe de liaison autem, fasse encore partie de la phrase initiale qu'il récapitule ${ }^{7}$. Quoi qu'il en soit, ce passage illustre la propension d'Albert à enfler indéfiniment sa phrase - ici par la surcharge de précisions et d'arguments nouveaux sur une structure initialement dialoguéejusqu'au risque de l'explosion grammaticale :

7 Nous nous écartons ici de la segmentation du texte de l'édition Santos Noya, qui fait d' « Ad haec autem non est difficile respondere » le début d'un nouveau paragraphe et comprend donc « tunc relatio universalis ad multa... » comme formant la principale de «Si dicatur quod habet illud in efficiente », comme si ce membre de phrase inaugurait déjà la réponse à l'objection. 


\section{Si dicatur}

quod habet illud in efficiente,

tunc universale non habet esse nisi ideale,

et cum praedicatur de singulari,

non praedicatur nisi sicut idea...

- Haec autem praedicatio non est substantialis, sed exemplaris tantum,

et sic destruitur omnis praedicatio substantialis.

$\mathrm{Si}$ autem habet illud in anima apprehendentis,

tunc relatio universalis ad multa non est nisi in anima et non in re,

et sic praedicatur nomine et non re

et sic universalia non sunt res, sed nomina,

quod falsum iam esse OSTENSUM EST.

Relinquitur ergo quod habeat illud secundum quod est res in se existens et $\mathrm{ab}$ agente et a cognoscente et a singulari separatum.

Ergo esse universalis est separatum ab esse singularis, et e converso.

Ex omnibus autem his et similibus videtur quod universalis esse separatum sit substantia, loco et tempore ab esse singularis -

Ad haec autem non est difficile respondere

ei qui bene novit positionem Peripateticorum:

per distinctionem esse universalis omnia haec facile determinantur.

Super Porphyrium, II, 5, éd. SANTOS NoYA, p. 31, 50-68.

Parfois le souci d'Albert d'articuler l'objection et sa réfutation va jusqu'à ce résultat surprenant, qu'il étoffe beaucoup plus la première que la seconde et semble ainsi s'intéresser plus à la thèse qu'il combat qu'à celle qu'il adopte finalement. Ainsi du passage suivant : soulevant et déterminant dans la même phrase la question de savoir s'il faut ou non aborder un sujet qui dépasse les forces de la logique, Albert est beaucoup plus prolixe dans la protase, qui présente les raisons de se taire, que dans l'apodose, qui expédie les raisons de parler, alors que c'est en sa faveur des secondes qu'il se décide finalement. Il y a donc comme précédemment une volonté de tout embrasser, les thèses avec leurs antithèses, mais qui manquer privilégier la thèse qu'il combat au détriment de la thèse qu'il défend: sans doute parce qu'il ne veut pas abandonner la première avant de l'avoir écoutée jusqu'au bout, comme pour lui donner toutes ses chances. Rien ne doit être méprisé.

Quamvis autem haec determinare sit supra vires logicae,

eo quod oportet in his uti rationibus sumptis ex principiis entis secundum quod est ens,

ex quibus cognoscitur

quid <sit> subsistens in intellectu solo, nudo et puro,

et aliam subsistentiam non habet,

et quid est subsistens extra intellectum in natura secundum esse completum,

- ad quod non sufficiunt rationes logicae -,

tamen propter bonitatem doctrinae,

et ne suspensus <sit> lectoris animus,

haec aliquantulum explanare temptandum est.

Super Porphyrium, II, 3, éd. SANTOS NoYA, p. 20, 61-69.

\section{Phrase «entonnoir»}

Également divisée en protase et apodose, un autre type de phrase, particulièrement caractéristique d'Albert et d'auteurs ultérieurs qu'il a influencés, pourrait être surnommée la phrase « entonnoir ». La première partie est la plus volumineuse : elle se compose d'un groupe complexe de subordonnées ; la seconde partie contient la principale et tire la conclusion de tous les éléments précédents. Sa forme typique est donc : «Étant donné que..., alors... »; si ce n'est que l' «Étant donné que... » se démultiple en «Étant donné que d'une part... et que, d'autre part, ou bien... ou 
bien... ou bien... ». Cette phrase entonnoir marque, encore une fois, un esprit encyclopédique et syncrétique, qui s'intéresse moins aux éléments séparés qu'à l'équilibre qu'ils forment tous réunis, qui engrange donc des données de toute nature et de toute origine, mais s'efforce ensuite, ou plutôt dans le même temps, d'en construire la synthèse.

Cum enim diffinitio dicens quid et propter quid medium sit in philosophia,

et diffinitio non possit variari nisi tripliciter,

scilicet quod aut concipiat materiam sensibilem,

quae cum motu est et mutatione

aut concipiat materiam intelligibilem,

cuius essentia non cum motu est et mutatione secundum rationem,

quamvis secundum esse sit in materia, quae est cum mutatione et motu,

sicut est magnitudo et numerus ;

aut nec secundum essentiam neque secundum esse concipit materiam sensibilem

neque secundum essentiam,

videtur quod philosophia non habeat nisi tres partes essentiales,

sicut et ARISTOTELES in VI PRIMAE PHILOSOPHIAE dicere videtur ;

propter quod nonnulli logicam sive rationalem nullam partem dicunt esse philosophiae.

Super Porphyrium, I, 2, éd. SANTOS NoyA, p. 2, 51-3, 3.

Si enim bonum et felicitas hominis est secundum optimae partis animae hominis perfectissimum actum,

hoc est secundum intellectum contemplativum,

nec contemplari poterit intellectus,

nisi noverit contemplationis principia,

et sciat invenire quod quaerit contemplari,

et diiudicare id ipsum quod iam contemplatur inventum,

patet quod prae omnibus utilis est ad felicitatem haec scientia

sine qua attingitur felicitatis actus

et per quam ipse felix actum non impeditae recipit operationis.

Super Porphyrium, I, 3, éd. SANTOS NOYA, p. 6, 17-25.

Voyons d'un peu plus près un cas intéressant de phrase entonnoir. La proposition principale en apodose contient la conclusion d'un raisonnement dont les prémisses se répartissent d'une façon plutôt désordonnée entre les quatre propositions subordonnées qui précèdent :

Cum enim productum producentis intelligentiae et agentis sit terminus,

- et producere est agere per intellectum -,

terminus autem actionis sit compositum et hoc aliquid,

cum universale producitur,

videtur quod universale sit compositum et hoc aliquid.

Super Porphyrium, II, 7, éd. SANTOS NoYA, p. 36, 16-20.

La thèse qu'Albert propose ici (avant de la réfuter plus loin) est que l'universel est à la fois un « composé » et un «ce-quelque chose » (hoc aliquid). Pour y arriver, il trace un chemin en quatre étapes, correspondant à quatre notions :
a) l'universel
b) le «produit », ici « de l'intelligence »
c) le « terme de l'action »
d) le «composé » et le « ce-quelque chose ». 
Au plan logique, le raisonnement reconstitué d'Albert consiste à passer de $a$ à $b$, puis de $b$ à $c$ et pour finir de $c$ à $d$ : l'universel est un produit, tout produit est le terme d'une action, tout terme d'une action est un composé et un «ce-quelque chose».

Toutefois, Albert n'a pas suivi cette voie, mais s'en est écarté de deux manières. D'abord, au lieu de dérouler comme un tapis rouge la série $a b c d$, il présente les choses dans l'ordre inattendu : de $b$ à $c$, de $c$ à $d$, de $a$ à $b$, donc de $a$ à $d$. Puisque le produit est le terme d'une action, que le terme d'une action est un composé et un ce-quelque chose, puisque l'universel est un produit, l'universel est (ou du moins semble être) un composé et un ce-quelque chose.

Albert a donc en tête un schéma logique correspondant à la figure rhétorique du climax ou de la gradatio, c'est-à-dire d'une démarche progressive, échelonnée; mais dans son discours il lui substitue un schéma grammatical plus complexe. Pourquoi ? Il y a deux raisons à cela. D'abord, il veut manifestement faire tenir tout le raisonnement dans une seule phrase, pressé qu'il est de tout englober, de tout intégrer, de tout concilier; ensuite, il veut faire correspondre la proposition principale qui, elle, est claire et directe, avec la conclusion de ce raisonnement. Comme si la principale, placée à la fin, était l'endroit d'une broderie dont les subordonnées seraient l'envers, c'est-à-dire cette face technique du travail qui admet toutes les bizarreries et irrégularités, puisqu'en fin de compte ce n'est pas sur elle que les regards se porteront. Les subordonnées sont en quelque sorte le chantier de la principale.

Dans ces subordonnées, outre l'ordre brisé qu'on a dit, il existe une seconde anomalie : c'est qu'Albert semble être revenu sur un premier raisonnement plus simple, pour le compliquer. En effet, si l'on s'en tient à ce qu'il écrit, le raisonnement suivant suffisait : tout universel est un produit, tout produit est le terme d'une action, tout terme d'une action est un «composé » et un «ce-quelque-chose ». Or ce raisonnement, logiquement suffisant, a été surchargé pour insister sur deux notions supplémentaires, qui à vrai dire n'étaient pas indispensables puisqu'elles sont déjà sous-entendues : celle d' " intelligence » et celle d' "agir ». En effet, dès lors qu'on a la notion de "produit », celles d'agir et d'intelligence sont déjà présentes, puisque, nous dit Albert, " produire, c'est agir par l'intelligence »; mais Albert a cru bon de faire un sort particulier à ces deux notions, de les tirer de l'implicite et de les inclure, comme une phase supplémentaire, à l'intérieur de son raisonnement. Pourquoi ? Parce que dans ce raisonnement il est conduit à parler du «terme d'une action». Dans «terme d'une action», il y a «terme», mais il y a aussi «action». Pour être complet, non pas complet logiquement, mais littéralement, il choisit donc d'ajouter à son raisonnement une proposition surérogatoire sur l'agir, ce qui le conduit à ajouter en plus des précisions sur l'intelligence, puisque ce qui distingue le produire des autres formes d'agir, c'est d'être un agir de l'intelligence. De fil en aiguille, il s'accule lui-même à cette tautologie initiale que «le produit est le terme d'une intelligence productrice et agente », alors que toute production, nous dit-il, est par définition l'action d'une intelligence. Albert a donc écrit cette phrase comme s'il s'était fait - au moment même ou plus tard - son propre exégète ; comme s'il n'avait rien voulu laisser passer sans le justifier absolument, même le superflu.

\section{Phrase décalée}

Une des difficultés les plus fréquentes que soulève la phrase d'Albert, c'est que bien souvent sa structure grammaticale est en décalage par rapport à sa structure logique. Prenons le passage suivant. Voulant dire que l'usage de la logique, tout en étant inscrit dans la nature de l'homme, a néanmoins besoin d'être développé par l'art, Albert donne la liste des opérations intellectuelles qui s'enchaînent pour aboutir à la connaissance de l'inconnu. Il en distingue quatre : la composition d'une forme avec une autre ou leur division; l'étonnement; l'enquête ; la confrontation des données recueillies. Avant ces quatre opérations, il y a l'homme et sa nature intellectuelle ; après 
elles, il y a la connaissance de l'inconnu. Tout ceci forme donc, logiquement, une échelle à quatre, et même à six barreaux, la pointe de l'extrait portant sur les passages successifs d'un échelon à l'autre :

1. L'homme est doué d'intellect;

2. étant doué d'intellect, il compose les formes entre elles ;

3. composant les formes, il s'étonne de ce qu'il comprend;

4. s'étonnant, il enquête ;

5. enquêtant, il confronte ;

6. confrontant, il parvient à la connaissance de l'inconnu.

On devrait donc avoir autant de phrases, ou, si on a l'esprit synthétique comme Albert, une seule phrase suffirait, composée de six membres ou propositions toutes sur le même plan. Au lieu de cela, les six étapes de la progression sont distribuées selon une division bipartite. D'abord un groupe de subordonnées, introduit par Ex hoc enim, comportant les deux premiers échelons ; ensuite un groupe de quatre propositions principales (ou indépendantes), construites sur le même type et commençant toutes par per : elles renferment les quatre échelons suivants. Bref, le processus d'acquisition des connaissances est clivé par une division bifide, qui ne semble pas avoir d'autre origine que l'habitude de composer des longues phrases à protase et apodose. Pourtant, il ne faudrait pas grand chose pour rapprocher la structure grammaticale de la structure logique, car déjà dans le groupe de subordonnées on retrouve un schéma comparable à celui des quatre principales : per intellectum compositivus est... Visiblement, Albert a commencé sa phrase, et c'est progressivement qu'a surgi dans son esprit un schéma plus simple, mieux adapté à ce qu'il veut dire. Un écrivain plus soigneux aurait repris le début de la phrase ; mais notre auteur ne se soucie, ni bien sûr de l'élégance, ni même de la transparence ou de la simple clarté de son style. Absorbé par l'enchaînement des idées, il marque une cruelle indifférence pour son discours, alors même qu'il réfléchit sur le langage, et semble persuadé que les phrases, tels des chats, finissent toujours par retomber sur leurs pattes.

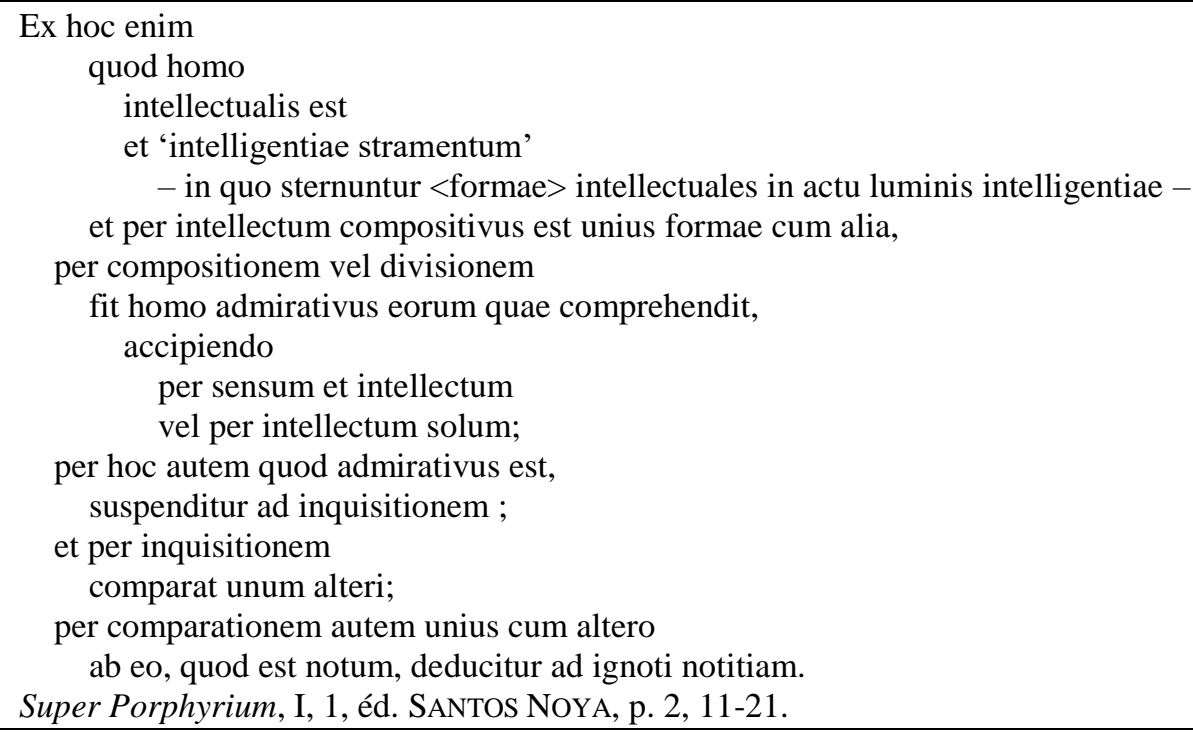

Dans la phrase suivante, Albert veut dire que la logique, dans la mesure où elle examine les relations topiques entre objets de connaissance, est indispensable pour chercher, pour trouver et pour savoir qu'on a trouvé ou non. Cette fois, la structure grammaticale correspond à la structure logique des idées à exprimer. À ceci près que la protase s'est démesurément enflée, parce qu'en parlant des relations «topiques », Albert n'a pu s'empêcher d'ouvrir une double parenthèse sur cet adjectif et son étymologie, puis sur les cinq types d'acte de connaissance par lesquels on les établit. 
Ce faisant, il s'est écarté de son but initial, mais qu'importe : insérant un complément qui récapitule la protase, ou plutôt ce qu'il aurait dû y dire (sine logica hoc docente), le voici ramené sur sa route et prêt à continuer la phrase jusqu'au bout. C'est là un exemple de phrase à deux pointes : la première que notre auteur réserve pour la fin, et qu'il n'oublie pas, même si, en chemin, il lui vient une seconde préoccupation, plus ponctuelle, celle d'expliquer une des notions dont il se sert. Comme il a l'esprit tendu vers l'idée principale, fidèlement conservée dans un coin de son esprit, il ne se résout pas à interrompre sa phrase. À défaut d'une pause, il fait donc une parenthèse. Au fond, la précision sur le mot topicae, étant de l'ordre des moyens, ne mérite pas à ses yeux une phrase pour elle seule ; car, on l'a compris, la phrase d'Albert est avant tout un mouvement, un élan, une tension, et sa longueur chronique reflète un effort permanent pour embrasser le plus d'éléments possible du réel, ou du connaissable.

Adhuc autem

per hoc quod inveniendi quodlibet scitum scientia est

per habitudines unius ad alterum

- quae topicae sive locales vocantur,

quibus intellectus unius

vel opinio

vel fides

vel existimatio

vel suspicio

locatur in alio,

quod iam intus habetur in animo quaerentis -

sine logica hoc docente

nec ad inquirendum

nec ad inveniendum aliquid

procedi potest,

nec etiam

quando quaesitum invenitur,

sciri potest,

an inventum

vel non inventum sit.

Manifestum est igitur quod, etiam quoad hoc, logica non tantum utilis sed necessaria etiam est ad omne philosophiam.

Super Porphyrium, I, 3, éd. SANTOS NoYA, p. 5, 63-71.

\section{CONCLUSION}

Que conclure de ce parcours dans les textes d'Albert, à fleur de phrases ? Ceci surtout : si notre auteur les allonge, c'est, pour une raison très simple : il veut tout dire à la fois. Il y a chez lui comme un monadisme grammatical : l'étude d'un objet particulier tend toujours à se déployer en considérations universelles, comme si le sort du tout était contenu dans celui de l'élément. Quelque objet qu'il examine, Albert a l'esprit occupé, non seulement par la question précise dont il traite, mais encore par tout le reste, c'est-à-dire tout ce dont il ne traite pas, mais qui est à comme à l'arrière-plan de son attention, et qui ne demande qu'à ressurgir dans son discours à la moindre occasion, car le souci primordial d'Albert, c'est d'organiser, de synthétiser, de hiérarchiser l'ensemble du connaissable.

De là son goût pour les phrases complexes, arborescentes, qui s'efforcent périodiquement de situer le point en question dans un tableau aussi complet que possible du savoir. Chaque phrase 
d'Albert tend, s'il n'y prend garde, à se transformer en un résumé de toute sa philosophie, de toute sa pensée. Là-dessus, il est possible que l'influence dionysienne ait amplifié une propension à « hiérarchiser » le réel, en le rangeant dans des arborescences indéfinies. Formellement en tous cas, la phrase omnivore d'Albert est en harmonie avec sa tension constante entre l'un et le multiple, avec sa métaphysique de l'illumination, avec ce mouvement par lequel la cause première se déploie hors d'elle-même pour produire l'univers. Cette phrase est donc cohérente avec la tentative d'Albert pour engranger tous les savoirs, pour s'abreuver à toutes les sources, et pour en faire une synthèse remarquablement ingénieuse, au risque de concilier des doctrines que nous savons radicalement incompatibles. En ce sens, la différence entre les phrases d'Albert et de Thomas est révélatrice de la différence entre leurs entreprises intellectuelles, malgré les parentés fortes que créent entre eux l'appartenance à un même ordre, celui des prêcheurs, et une relation de maître à disciple. Revenons donc sur notre comparaison initiale.

\begin{tabular}{|c|c|}
\hline $\begin{array}{l}\text { Albert le Grand, } \\
\text { Summa theologiae, tr. 1, qu. 3, a. } 2\end{array}$ & $\begin{array}{c}\text { Thomas d'Aquin, } \\
\text { Summa theologiae, } \mathrm{I}^{\mathrm{a}} \text {, qu. } 1, \text { a. } 3\end{array}$ \\
\hline $\begin{array}{l}\text { Iuxta hoc quaeritur secundo, utrum theologia sit una vel } \\
\text { plures, et qua unitate sit una si una est. } \\
\text { Videtur enim quod non sit una. } \\
\text { Dicit enim Aristoteles in I PosTERIORUM quod una est } \\
\text { scientia quae est unius generis subiecti. } \\
\text { Et est sensus } \\
\text { quod una scientia est de uno subiecto in genere } \\
\text { ad cuius ambitum praedicationis reducuntur omnia } \\
\text { quae sunt sicut partes in totalitate subiecti, } \\
\text { sicut geometria de quantitate continua est, } \\
\text { ad cuius communitatem omnia reducuntur } \\
\text { quae considerat geometria ; } \\
\text { et sic arithmetica una scientia est, } \\
\text { quae est de numeris. } \\
\text { Et non tantum hoc est in realibus, sed etiam in } \\
\text { quae de syllogismo est et partibus syllogismi, } \\
\text { ad cuius formam omnes aliae ratiocinationes } \\
\text { logicis, } \\
\text { reducuntur. } \quad \text { autem tale commune res et signa reduci non } \\
\text { Ad unum } \\
\text { possunt, } \\
\text { nec Christus et opera redemptionis. } \\
\text { Crucifixio enim et mors passiones sunt, } \\
\text { resurrectio vero et ascensio actiones ; } \\
\text { actio vero et passio ad unum commune genus non reducuntur. } \\
\text { Similiter caput et corpus et actiones capitis et corporis in uno } \\
\text { communi genere non continentur cum passionibus eorundem. }\end{array}$ & $\begin{array}{l}\text { Ad tertium sic proceditur. } \\
\text { Videtur quod sacra doctrina non sit una } \\
\text { scientia } \\
\text { quia, secundum Philosophum in I } \\
\text { PosTERIORUM, una scientia est quae est unius } \\
\text { generis subiecti. }\end{array}$ \\
\hline
\end{tabular}

À droite, Thomas exprime son argument avec une grande sobriété de mots et de concepts, sous la forme d'un syllogisme. Après l'énoncé de la thèse, on distingue une majeure, formée par la citation d'Aristote (l'unité d'une science implique l'unité générique de son sujet); puis une mineure (or la doctrine sacrée traite du créateur et de la créature, qui ne sont pas du même genre); enfin la conclusion (donc la doctrine sacrée n'est pas une science « une »). En face, Albert utilise la même citation, parvient à la même conclusion, mais entre les deux il a fait deux longs détours. D'abord, expliquant la phrase d'Aristote, il a multiplié les exemples extérieurs à la doctrine sacrée : dans la géométrie, dans l'arithmétique, dans la logique ; le tout en décrivant méthodiquement ce sur quoi porte chacune de ces sciences. Il s'arrête certes avant d'avoir donné une division complète du 
savoir, mais on voit bien que la tentation n'était pas loin. Plus loin, voulant montrer que la doctrine sacrée porte sur des objets hétérogènes, il ne s'arrête pas un exemple unique, le plus flagrant, comme Thomas (le couple créateur / créature) ; mais, une fois encore, il multiplie les exemples en énumérant plusieurs couples : choses / signes ; Christ / œuvres de rédemption ; passions / actions ; tête / corps. Ces deux dernières oppositions se croisent d'ailleurs pour obtenir quatre réalités : actions de la tête / passions de la tête / actions du corps / passions du corps. Par là, il esquisse une division complète de la théologie, là où l'on se demandait simplement si elle était une ; mais ce dérapage apparent est logique, puisque pour Albert l'unité d'une science se mesure à son aptitude à embrasser l'universalité de son sujet.

1) Albert

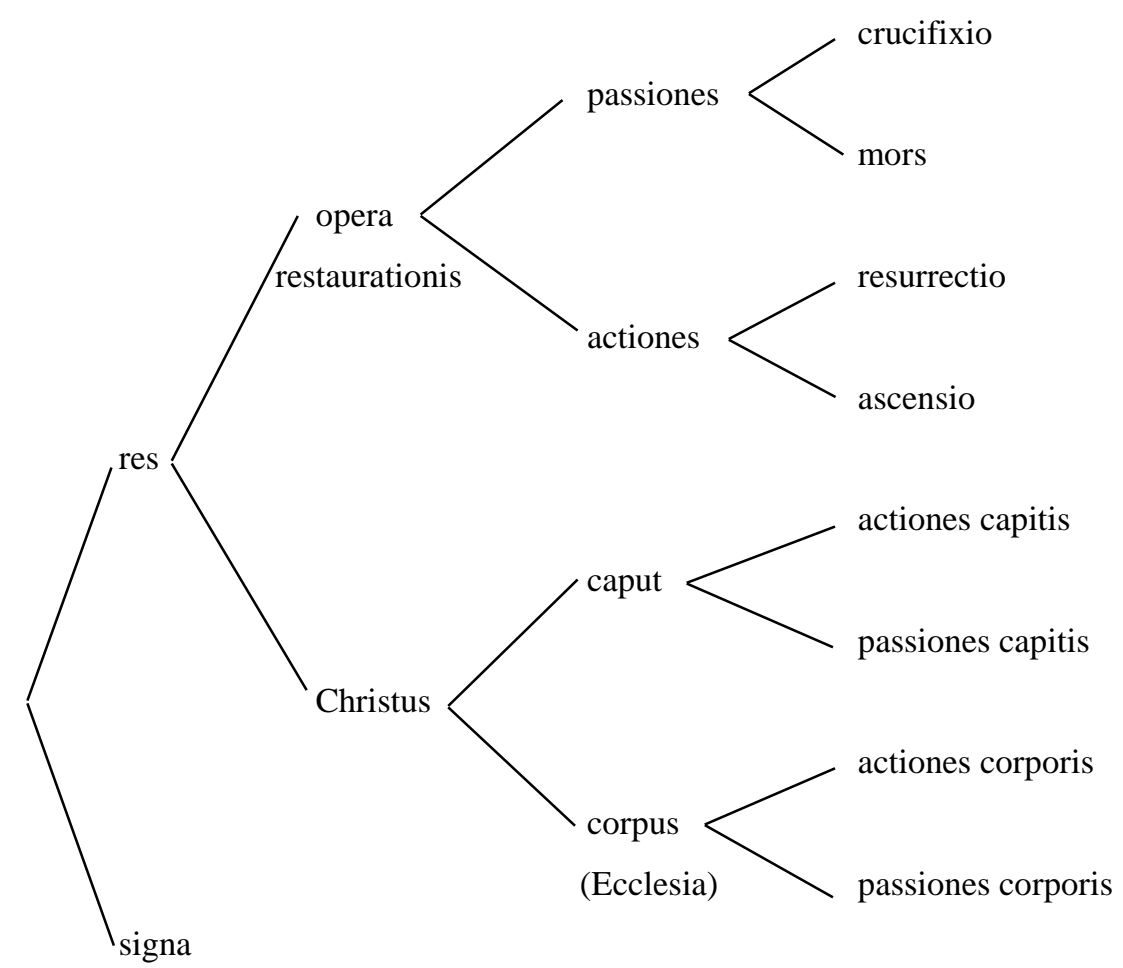

2) Thomas

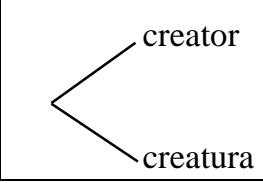

La comparaison est donc instructive. Albert et Thomas ont la même visée : tous deux veulent édifier une synthèse philosophique et théologique aussi achevée que possible. Mais alors que pour Thomas cette synthèse est acquise, moyennant certains choix - Aristote de préférence aux autres philosophes - pour Albert la synthèse est une œuvre en progrès, un projet en cours de réalisation, d'abord parce qu'il veut tout embrasser, tout rassembler, tout concilier y compris l'inconciliable, ensuite parce que le travail de l'écriture n'est pas distinct chez celui de celui de la pensée : écrivain à plume chercheuse, Albert associe le lecteur à son enquête, à ses hésitations et à ses bifurcations, à sa confiance aussi dans la rationalité du monde et du connaissable. Tôt ou tard et fût-ce cahin-caha, 
sa phrase et la raison, parie-t-il, finiront bien par retomber sur leurs pieds. Là donc où Thomas, calmement, pose, telles des briques homogènes, ses phrases l'une à côté de l'autre dans un bâtiment dont il a en tête les plans bien arrêtés, Albert, parce que c'est son tempérament, peut-être aussi parce qu'il vient avant Thomas, semble à chaque instant recalculer et revérifier la taille, la forme et la solidité de l'édifice total, au moment même où il en équarrit et en ajuste les moellons colossaux et irréguliers. En somme, si la phrase d'Albert est interminable, c'est d'abord parce que sa synthèse est inachevée. 